REVISTA ANDALUZA DE ANTROPOLOGÍA.

NÚMERO 4: ENCRUCIJADAS PARA LAS SOCIEDADES PESQUERAS DEL SUR EN EL MARCO DE LA GLOBALIZACIÓN. MARZO DE 2013

ISSN 2174-6796

[pp. 122-146]

http://dx.doi.org/10.12795/RAA.2013.i04.07

Fecha de recepción: 13/11/2012

Fecha de aceptación: 21/12/2012

\title{
CAPITALISMO PESQUERO Y SINDICALISMO EN LA ESPAÑA DE ANTEGUERRA: LA FEDERACIÓN NACIONAL DE INDUSTRIA PESQUERA*
}

\author{
Dionisio Pereira González \\ Universidad de Santiago de Compostela
}

\section{Resumen.}

Este trabajo tiene por objetivo enmarcar la particular singladura de la Federación Nacional de Obreros de la Industria Pesquera y sus Derivados (FNIP) incorporada a la CNT (1931), tomando como referente organizativo dentro de la central anarcosindicalista a la Federación Regional Galaica de Industria Pesquera y sus Derivados (FRGIP), que la precedió en el tiempo, y a Galicia, principal zona pesquera, como referente territorial. Y decimos particular en lo orgánico, porque fue la federación de industria de la Confederación que más trató de desarrollar una visión de ramo superadora de los gremios, incluyendo tanto a pescadores como a las trabajadoras de la conserva, a los autopatronos transportistas de pescado y, con ciertas condiciones, a los pequeños armadores. La aparición e implantación mayoritaria de la FRGIP en Galicia, un territorio periférico alejado de las grandes concentraciones proletarias del Estado español y con una composición social específica, confieren, asimismo, una llamativa singularidad al sindicalismo pesquero de clase en la costa gallega y española.

Palabras clave. Pesca, Antagonismo, Conflictividad, Galicia, Patronal, Anarcosindicalismo, CNT.

\footnotetext{
* Este trabajo se inscribe dentro del proyecto de investigación del Plan Nacional de Investigación I+D+i Patrimonio Etnológico, Sociedad y Cultura Marítima, PESCUM (HAR 2010-15566), financiado por el Ministerio de Economía y Competitividad (2010-2013)
} 


\begin{abstract}
.
This work aims to contextualize the particular pathway of the Fishing Industry and their Derivatives Workers National Federation (FNIP), built-in to the CNT (1931). The Galician Regional Federation of the Fishing Industry and their Derivatives (FRGIP), within the anarcho-syndicalist central, is taken as a benchmark. At the same time, we take into account Galicia as the main fishing area at the Spanish frame. This FishingMaritime Union tried to develop a sectoral vision surpassing the guilds, including both fishermen, women of the processing industry, the autoemployers of fisheries transport, such as small boat owners - these with certain conditions. The emergence and majority implantation of the FRGIP in Galicia, a peripheral territory away from the great proletarian concentrations of the Spanish State and with a specific social composition, confers also a striking singularity to fishing class trade unionism in Galician and Spanish coast.
\end{abstract}

\title{
KeyWords
}

Fishing, Antagonism, Conflicts, Galicia, Employer's Fisheries, Anarcho-syndicalism, Confederación Nacional del Trabajo.

\section{INTRODUCCIÓN: DE LA PESCA ARTESANAL AL CAPITALISMO PESQUERO}

La conciencia de la dura condición del pescador estará en el instinto asociativo que, avanzando el siglo XIX, entronca con los Gremios de Mar medievales. Después, el asociacionismo marinero desenvuelto en el primer tercio del siglo pasado, va a progresar en un panorama cambiante en cuanto a las actividades pesqueras. Como se sabe (Giráldez, 1989), en el transcurso de este período, los pescadores gallegos pasan de faenar estacionalmente a pocas millas de la costa procurando mayormente sardina y merluza con artes tradicionales y a bordo de pequeñas embarcaciones impulsadas a vela y remo de propiedad familiar, a tripular una flota de vapores equipados con artes intensivas, que, tras esquilmar los bancos de pesca en el litoral, frecuentaba caladeros distantes y atendía las crecientes necesidades urbanas de un consumo masivo de pescado fresco. En realidad, se estaba produciendo un cambio estructural en el sector, derivado de la transformación del modo de producción pesquero: en aquel tiempo se vivió la transición entre la pesca artesanal y el capitalismo pesquero, inducido este bien por el desarrollo acelerado de las actividades de transformación, verdaderas dominadoras del mercado de la sardina, bien por la demanda urbana de pescado de mesa alentada por la mejora de las comunicaciones.

Tomando como punto de referencia las estadísticas oficiales, en 1932 la flota gallega estaba compuesta por 14.161 embarcaciones, que desplazaban 60.000 TRB, capturaban 127.000 Tns. de pescado y tenían como dotación 58.000 hombres. Por aquellos días se evidenciaba 
un innegable avance tecnológico, pues el 70\% del tonelaje y el 49\% de las tripulaciones correspondía ya a barcos de propulsión mecánica, progresivamente concentrados en los puertos de Vigo-Bouzas, Coruña y Marín, principales centros de aprovisionamiento, de las descargas de pescado y de la contratación del personal, procedente en buena medida de pequeñas villas marineras (Pereira, 1992). A "grosso modo", la flota pesquera gallega representaba la tercera parte de la española en cuanto a buques y tripulantes (Ministerio Industria, 1933).

En la costa, no obstante, convivirán hasta hoy los dos modelos pesqueros: el de la pesca artesanal, vinculada al espacio familiar, y lo que podríamos denominar de transición a la pesca industrial o al capitalismo pesquero, ejemplarizada en la diversidad de actividades extractivas y sus derivadas del puerto de Vigo-Bouzas, única experiencia industrial estructurada en la Galicia de anteguerra. Esta intersección de modelos daba lugar a la frecuente fusión de los contornos clasistas de los pescadores, pues no era extraño encontrar tripulantes enrolados en las motoras sardineras o en los vapores de arrastre que, durante el invierno, cambiaban de actividad y andaban en el interior de las rías a los llamados "oficios baixos" en pequeñas embarcaciones de su propiedad (Pereira, 2002); también era (y es) frecuente que un pescador asalariado de la flota industrial acabase su vida laboral en la pesca artesanal como pequeño armador (Vázquez, 1990; Candía y Ribeiro, 1989). La persistencia de las pescas artesanales ofrecía, además, una alternativa para los tripulantes de la pesca industrial o semi-industrial en el caso de que sus actividades se interrumpieran por cualquier circunstancia (Martínez, 1989; García, 1989). Toda esta realidad, compleja, unida a la no menos habitual condición de propietarios o arrendatarios de pequeñas parcelas en el litoral (Giráldez, 1996; Calo, 1978; García, 1989), estará, como veremos, muy presente en las estrategias de los marineros en conflicto.

Como se sabe, en lo tocante al modelo artesanal el objetivo fundamental de la pesca era abastecer las necesidades familiares, empleando una tecnología tradicional para faenar en caladeros próximos a la costa, con unas relaciones de producción definidas por un mitigado antagonismo entre patrones/armadores y marineros, que compartían los trabajos extractivos y una distribución relativamente equitativa del producto (Delgado, 1998). Por el contrario, el incipiente capitalismo pesquero desarrollado desde finales del siglo XIX en los principales puertos de pesca españoles (Vigo-Bouzas, Pasaia, Coruña, Gijón, Cádiz, Algeciras, Barcelona, Santander, Marín, por este orden), tenía como finalidad la maximización de beneficios para amortizar unas voluminosas inversiones, que posibilitaban la introducción de barcos con propulsión mecánica y unas técnicas que intensificaban las capturas en zonas de pesca cada vez más lejanas. En las embarcaciones, además, se desarrollaron unas nuevas relaciones sociolaborales caracterizadas por un mayor antagonismo y desigualdad, simbolizadas tanto por el control de los nuevos 
medios de producción en pocas manos, como por el endurecimiento de las condiciones de trabajo y un reparto del producto menos equitativa que en la pesca artesanal (González, 1977).

Se puede decir que, en la costa gallega, el tránsito del modo de producción artesanal a un capitalismo pesquero más o menos desarrollado, fue visible en muchas villas costeras en torno a la I ${ }^{\text {a }}$ Guerra Mundial. Al igual de lo que ocurriera entre siglos en los tres grandes puertos pesqueros, ahora en Cangas, Moaña, Bueu, Baiona, Cariño, Celeiro o Ribeira, se observaba cómo los tripulantes de las embarcaciones tradicionales eran absorbidos por los vapores y motoras, y también cómo la pesca con los aparejos artesanales era sustituida por las capturas masivas de los cercos, los palangres de fondo y el arrastre con el "bou" o en pareja. Como decimos, en las nuevas embarcaciones los tripulantes soportaron unas relaciones de producción bien distintas del paternalismo propio del Antiguo Régimen ${ }^{1}$. De inicio, muchos armadores que concentraban dotaciones de 30 o 40 hombres en sus vapores y motoras dejaron de ir al mar, quedando escindido el colectivo pesquero. Además, los trabajos de la pesca intensiva se hicieron más penosos en las, a partir de ahora, largas mareas, ya que no había correspondencia entre las grandes dimensiones de las nuevas artes y el mayor volumen de las capturas, con la pequeña potencia de los primeros dispositivos mecánicos de virada. Entonces, la única solución era incorporar más brazos a bordo, agravándose las condiciones de habitabilidad, ya espartanas, de los buques.

En el nuevo modelo pesquero las tareas de tierra requirieron, también, más tiempo, mayor esfuerzo y, por consiguiente, demandaron mejor retribución. Fijémonos en Marín; allí los vapores pareja de arrastre del día faenaban 14 horas y había que contar, a mayores, con las labores de tierra: descargar los pesados aparejos y teñirlos cada 15 días, lavar las redes cada dos o tres, acarrear agua, palear carbón y acomodarlo en las carboneras, reparar los aparejos.... En 1910 no amarraban ni los domingos: la bajada de los barómetros y las fiestas patronales decidían las recaladas. El pago de tantos trabajos era de 90 pts./ mes, sin que las faenas de tierra merecieran ningún pago (Maná, 1994).

En algunos barcos la manera de retribuir también cambió: en los "bous" y en las parejas de arrastre se introdujo a comienzos del siglo XX el pago a jornal, diferenciado entre los marineros, el personal de máquina y los patrones. En el resto de los oficios persistió el tradicional reparto en "quiñones", si bien, ahora, en las motoras sardineras, el porcentaje a percibir fue tanto más favorable al armador conforme el capital invertido en la embarcación y en los aparejos fuese mayor. A esta desigualdad en el reparto (Calo,

1. Aunque hay quien idealiza el paternalismo igualitariod esenvuelto en la "cristiana y equitativa economía comunal de los cercos" gremiales, consta ela n tagonismo social ya en los Gremios de Pescadores medievales (Filgueira, 1946: 13; Carmona, 1998). 
1996), se unirá la certeza de que los armadores pretenderán en adelante descontar las innovaciones introducidas en los barcos (las primeras maquinitas de vapor para virar, por ejemplo) de las partes de los tripulantes, o remolonear en el pago de las faenas en tierra, provocando que muchos marineros no retribuidos a jornal tomasen conciencia de su condición de desposeídos, a pesar de no estar sujetos al salario (Pereira, 2010).

Es de subrayar que, contrariamente a lo que sucedió en zonas de Euskadi (Bermeo, sin ir más lejos), donde los socialistas lastrados si acaso por una rígida aplicación del ideario marxista renunciaron hasta los días de la II ${ }^{a}$ República a incorporar a los trabajadores de la pesca artesanal en las sociedades de resistencia, puesto que consideraban propietarios/ cooperativistas a los tripulantes no retribuidos a jornal fijo, en el litoral gallego, y haciendo gala de un notorio pragmatismo, los socialistas no tuvieron impedimento alguno para alentar, ya desde finales del siglo XIX, colectivos integrados por marineros y pequeños armadores que cobraban "a la parte". Esta circunstancia, unida a las "intersecciones" antedichas entre la pesca artesanal y la de transición al capitalismo pesquero, ayudó sobremanera al desarrollo del asociacionismo de clase en las villas marineras de las Rías Baixas gallegas después de la Ia Guerra Mundial.

La confrontación, pues, acompañó a la transición pesquera; una conflictividad que va a estar presente durante el período en todos los estadios del ciclo productivo, tendrá múltiples consecuencias en las relaciones sociales de los distintos puertos y será especialmente visible en la flota sardinera y en la pesca de arrastre. El proceso (conflictivo) de cambio también vino acompañado por la eclosión del asociacionismo en el sector: un asociacionismo de clase dividido en sus dos polos opuestos patronal y obrero, este último muy combativo y mayoritario en Galicia a la altura del 18 de julio de 1936, y un asociacionismo mixto representado en buena medida por los Pósitos de Pescadores, conciliador de las aspiraciones de armadores y tripulantes (Pereira, 2012). Pero, en cualquier caso, uno y otro representativos de los diversos segmentos del colectivo marinero, cada vez más conscientes de sus intereses y organizados para su defensa, en un proceso que llega a su cenit en la II República.

Ciertamente, la identidad de los propios pescadores también estuvo sujeta a un cambio profundo en este primer tercio de la pasada centuria, un período en cuyo final las fuerzas productivas asociadas a la pesca artesanal ya solo representaban la mitad del universo pesquero gallego. Aunque el trabajo de Benigno Rodríguez Santamaría, llevado a cabo en tanto que comisionado del Ministerio de Marina entre 1907 y 1910 y publicado más

2. En este aspecto, los socialistas vascos coincidían con visiones más bien conservadoras que deseaban separar a los marineros no retribuidos a jornal de los organismos de clase, caso del Senador del Reino Fermín Calbetón, de Darío de Areitio, Tomás Balbás, o del funcionario del Ministerio de Marina y sociólogo vocacional, Benigno Rodríguez Santamaría (Delgado, 1998). 
tarde en 1916, es de un gran valor sociológico, nos llama la atención el hecho de que el autor ignorara el importante desarrollo asociativo que, en el ámbito pesquero, tuvo lugar por aquel tiempo en Galicia tanto en cuanto a agrupaciones de carácter mixto, como de clase (Pereira, 2010). Además, para Rodríguez Santamaría un marinero a jornal no era más que un operario dependiente en todo de su armador, mal retribuido e imposibilitado para ejercer la profesión de pescador propiamente dicha, asimilando esta al protagonismo en la toma de decisiones en los trabajos del mar y a la propiedad de los medios de producción, por lo que aquel obrero/pescador no precisaba (en su opinión) de ningún tipo de asociacionismo propio de las faenas pesqueras (Rodríguez, 1916). Adelantándose Rodríguez Santamaría a posturas semejantes como la del catalán Emerenciá Roig (Roig, 1927) y la del vasco Fermín Calbetón, desde su punto de vista y sin tener en cuenta ni la tendencia al desarrollo acelerado del capitalismo pesquero en numerosos puertos, ni las actividades que ya no se podían conceptuar como tradicionales o artesanales (la pesca de la sardina con el cerco-jareta, primero desde las traineras y luego desde las motoras, por ejemplo), los proletarios, las sociedades de resistencia y la lucha de clases eran una "anomalía" en el mundo del mar, que se pretendía caracterizar por una idealizada mayoría social (los llamados por aquel entonces "pescadores libres") compuesta por pequeños propietarios individuales o asociados con otros, a los que habría que añadir los marineros sujetos a las "partijas" e incorporados a la pesca artesanal (Calbetón, 1918).

Una vez constatada la irrupción del capitalismo pesquero y del asociacionismo mixto o de clase en el mundo del mar, quizás habría que poner en cuestión ciertas caracterizaciones "esencialistas" vinculadas a la pesca artesanal de componente familiar, que en Galicia identificaron de manera inmutable el comportamiento de los pescadores con el localismo, el individualismo, la competencia, la mentira y la envidia, reduciendo el antagonismo a las confrontaciones con otras capas sociales como los campesinos y con los pescadores de puertos vecinos (Calo, 1996). A lo largo de nuestras investigaciones, dimos cuenta como, tras casi 70 años de trayectoria constructiva y conflictiva a un tiempo, los integrantes del asociacionismo marinero de anteguerra promovieron en el litoral gallego actitudes bien distintas, relacionadas con la solidaridad de clase supralocal, el apoyo mutuo, el cooperativismo, la ordenación de los recursos y el civismo democrático (Pereira, 2010). No en vano al sindicato confederal Fraternidad Marinera se le conoció durante la II ${ }^{\mathrm{a}}$ República como "o corazón de Moaña", por la importante labor de construcción de la ciudadanía y la extensión sociocultural que realizó en aquella populosa villa marinera (Pereira, 1994). En dialéctica reciprocidad, la progresiva extensión de estos valores durante el período considerado, retroalimentó el propio desarrollo asociativo. Todo ello quedó, naturalmente, en suspenso tras el brutal movimiento militar de 1936. Quede aquí tan sólo esbozada esta cuestión, que requeriría un mayor desarrollo. 


\section{GÉNESIS DE LA FEDERACIÓN REGIONAL GALAICA DE INDUSTRIA PESQUERA Y SUS DERIVADOS}

Contando con la hegemonía de los anarcosindicalistas integrados en la Federación Local Obrera de A Coruña, nacía en esta capital a finales de 1914 uno de los colectivos más longevos y representativos de la CNT herculina: la Sociedad de Marineros, Fogoneros y Patrones El Despertar Marítimo, que incorporaba a sus filas tanto los tripulantes de la Marina Mercante y de servicio de puerto, como las dotaciones retribuidas a jornal de los pesqueros.

Como se sabe, la visión sectorial del sindicalismo superadora de los oficios, fue aprobada en el Congreso de la CNT celebrado al final de 1919. Al socaire de las decisiones congresuales enfocadas hacia los sindicatos de industria, en el muelle corunés se consolidará esta nueva concepción sindical un año después, ya que El Despertar Marítimo se convertirá en Sección del Sindicato Único del Transporte Marítimo y Terrestre, que agrupaba todas las profesiones relacionadas con las tareas portuarias. Consciente de su poderío, manifestado en 1920 con el paro durante cinco meses de las parejas de arrastre que descargaban en Coruña, la organización herculina empezó a presionar a los tripulantes de aquellos arrastreros matriculados en Marín y Bouzas pero con base en Coruña, para que abandonasen la UGT y se incorporasen a la CNT, iniciando en aquellos dos puertos unas disputas que ni siquiera la Dictadura de Primo de Rivera pudo cortar.

Con la llegada del régimen autoritario, el clima represivo provocó el decaimiento del societarismo marinero en las pequeñas localidades costeras, sin que los sindicatos de clase dejaran de funcionar en los grandes puertos. Así, en el primer año de la Dictadura, se producirá una dura competencia por el control del puerto de Vigo-Bouzas entre el Sindicato General de Trabajadores (CNT) y el Comité Regional de Obreros de la Industria Pesquera de Galicia y Similares cercano a la UGT, conscientes cenetistas y socialistas de su importancia estratégica. Al final, la CNT impuso su mejor organización en el muelle coruñés, donde recalaban numerosas parejas de Bouzas, de manera que muchos asociados a la UGT en este último puerto solicitaron en el año 1925 su integración en la Sección Flota Pesquera del Sindicato General de Trabajadores, tras las gestiones del fogonero coruñés Manuel Montes, presidente a la sazón de El Despertar Marítimo ${ }^{3}$.

Desaparecido el Comité Regional a partir de 1925, los anarcosindicalistas articularon ese mismo año la Federación Regional Marítima, para agrupar todas las profesiones vinculadas al mar, contando con una fuerte influencia en los puertos de Coruña, VigoBouzas. La asamblea constituyente tuvo lugar en Compostela el día 6 de diciembre; a ella asistieron, entre otros, el entonces secretario de la Confederación Regional Galaica (CRG) de la CNT, Manuel Fandiño; el director del periódico Solidaridad Obrera, Ezequiel Rey;

3. olidaridad Obrera, Santiago, 29/8/1925. 
Manuel Montes, por El Despertar Marítimo y José Villaverde, por Vigo-Bouzas. Además del acuerdo para crear la Federación, tuvo importancia el debate sobre su carácter "nacional" o "regional". Al final, se adoptó la propuesta de Villaverde, consistente en que "...por el momento debe ser regional con aspiraciones de nacional, y creada la federación marítima gallega, con hechos se les puede demostrar a las demás regiones las ventajas que tiene la organización" ${ }^{4}$.

Con sede en Coruña, la referida Federación se legalizó al año siguiente bajo el rótulo de Federación Regional Galaica de la Industria Pesquera y sus Derivados (FRGIP) y tuvo durante toda la etapa de la Dictadura a Montes como secretario y a Villaverde, liberado de la Sección Flota Pesquera viguesa, como redactor de su vocero El Despertar Marítimo 5 . Culminaban así más de cinco años de trabajos para organizar la federación pesquera confederal a nivel galaico, pues la estrategia ya se anunciara en 1919 durante el Congreso de la CNT. La FRGIP fue, por tanto, precursora de la futura estructuración cenetista en federaciones de industria, aprobada seis años después en el Congreso de 1931 y también antecesora de la Federación Nacional de Obreros de la Industria Pesquera y sus Derivados (FNIP), conformada ese mismo año.

Así, la FRGIP pudo conservar durante la "Dictablanda" una mínima armazón en Coruña, sostenida por El Despertar Marítimo; por consiguiente, la CNT galaica contó con una estructura sindical en el mar que se demostraría muy útil, cuando la descomposición del régimen posibilitó la reorganización de la totalidad del sindicalismo marinero de clase.

Paralelamente al itinerario recorrido por sus antagonistas, la estrategia de los armadores organizados fue procurar la expansión territorial y el reforzamiento de los distintos colectivos patronales, alentando organismos más allá del ámbito local, siempre desde una defensa numantina del derecho "natural" atribuido a los propietarios de los medios de producción sobre la admisión y el despido del personal en los barcos. Colectivos como la Asociación General de Industrias Pesqueras y Derivados, concebida en Vigo como "lobby" económico de ámbito galaico en octubre de 1917, tuvieron un papel principal en la superación del localismo por parte de los armadores y fabricantes organizados en la costa gallega. En concreto, con posterioridad a la Ia Guerra Mundial se dieron pasos de cara a una estructuración gallega de la Patronal pesquera, quizás para responder a las Federaciones regionales impulsadas por socialistas y libertarios: la constitución en Vigo bajo patrocinio de la susodicha Asociación del Sindicato de Armadores de Vapores Pesqueros de Galicia andando el mes de agosto de 1919, por ejemplo.

4. Oficio fechado el 30/12/1925; Fondo Goberno Civil de Coruña, Asociacións Coruña, Cp. El Despertar Marítimo, Arquivo do Reino de Galicia (ARG), A Coruña.

5. La Federación Regional Galaica de la Industria Pesquera y sus Derivados, figura inscrita en 1926, Fondo Goberno Civil de Coruña, Asociacións Coruña, Cp. G-2657/13, ARG. 
Una vez organizada siquiera de manera incipiente la Patronal pesquera galaica, la propia Asociación General ofició como referente ya desde aquel año para la creación de una Federación de Armadores de Buques de Pesca de España, consolidada en 1923 con las patronales de la pesca industrial de Barcelona, Pasaia, Donostia, Cádiz, Huelva, Málaga, Gijón, Vigo-Bouzas, Marín y Coruña. Paralelamente y también a partir de 1919, la Asociación impulsó la Federación Nacional de Industrias Pesqueras y sus Derivados, concebida como grupo de presión económico y político que incorporaba armadores, conserveros y salazoneros de 18 puertos españoles (Ministerio de Marina, 1920). Culminaba así una visión más estructurada y global de los intereses de clase de los principales propietarios de medios de producción en la industria pesquera a nivel estatal, conformando un interlocutor colectivo privilegiado para las negociaciones con la Administración en temas considerados clave para el futuro del sector ${ }^{6}$.

En lo sucesivo, durante el conflictivo tiempo republicano la fijación de las bases de trabajo con los sindicatos trascenderá en buena medida el marco local, para conformarse un espacio de negociación con ámbito estatal. En cualquier caso, y tanto en lo referido a la Patronal como a los sindicatos marineros, en este nuevo escenario supra local el protagonismo y el carácter pionero de los agentes sociales radicados en Galicia, será muy relevante.

\section{NUEVO IMPULSO}

En febrero de 1930 la tragedia arribó al puerto de Bouzas: cuatro vapores parejas con 42 tripulantes, se hundían en el Grand Sole. El luto obligó al amarre de la flota y la indignación avivó las conciencias adormecidas; aprovechando el ambiente, José Villaverde y Manuel Montes convocaron una asamblea para reorganizar la Sección Flota Pesquera del Sindicato General de Trabajadores de Vigo y, así mismo, relanzar la FRGIP .

Para consolidar la organización pesquera de Vigo, en la CRG se tomó una importante decisión: el traslado como liberado a la ciudad olívica de su antiguo secretario, el carpintero compostelano Manuel Fandiño, hombre de probada capacidad negociadora y organizativa; su cometido será el de asumir la secretaría de la FRGIP. Con los sindicatos marineros de Vigo, Marín y Coruña operando en las localidades que focalizaban la mayoría de las actividades pesqueras, la Regional Pesquera ya podía dejar sentir su peso en los pequeños puertos. Mas para ello, debía probar su fuerza frente a los armadores y, en un proceso paralelo, adaptar la estructuración interna a la compleja realidad económica y social de la pesca.

Para calibrar el proceso que estamos explicando, es preciso poner de manifiesto las

6. Actas del Consejo de Dirección de la Federación de Armadores de Buques de Pesca de España, Años 1925-1928, Archivo privado. También, Ministerio de Marina (1920).

7. ¡Despertad!, Vigo, 26/7/1930. 
dificultades para practicar una acción sindical estable en la pesca en general y en la de altura en particular, pues los buques, o pasaban muchos días lejos de tierra apartados unos de otros, o, en el caso de la bajura, la gente estaba en el mar la mayoría de la jornada, en horarios en absoluto equiparables a los de los trabajadores terrestres. Estas dificultades, por lo tanto, justificaban algunas medidas poco habituales en la CNT, como el empleo de liberados sindicales, figura normalmente muy discutida por los confederales, opuestos a la profesionalización del sindicalismo; actuando con pragmatismo y dejando fuera aspectos doctrinales, los liberados fueron admitidos en distintas ocasiones y también en diferentes puertos (Vigo, Coruña, Marín, Cariño, Ribeira,..) con la finalidad de que estuviesen en condiciones de atender los problemas que pudieran surgir en tierra en ausencia de los asociados. Hay que tener en cuenta que para realizar una asamblea donde poder discutir cuestiones de interés general, era necesario el amarre temporal de la flota pesquera en cuestión.

Tras el 14 de Abril de 1931 y por iniciativa del Ministerio de Marina, tendrán lugar a nivel estatal las negociaciones entre la Federación de Armadores de Buques de Pesca de España y los sindicatos UGT y CNT, iniciadas en Madrid durante aquel mes y prolongadas en distintas fases hasta la primavera de 1932, con las mejoras salariales, reglamentación del descanso, subsidio de enfermedad, incremento de las dotaciones y garantías en contrataciones y despidos, como temas planteados por los colectivos marineros. Paralelamente, se van a suceder las huelgas por estas motivaciones en las flotas de altura radicadas en Coruña, Ferrol, Gijón, Pasaia, Huelva o Málaga, de manera que los armadores tendrán la certeza de que había un movimiento de fondo que trascendía la problemática local ${ }^{8}$. Según José Villaverde, uno de los representantes en liza, las reuniones de Madrid fueron más que nada una tentativa de las autoridades republicanas de cara a establecer una corriente de armonía entre los sindicatos pesqueros y la Patronal, para desarrollar un nuevo marco de relaciones laborales. De acuerdo con el propio Villaverde, desde el comienzo la Patronal se mostró reacia al diálogo para la aprobación de unas bases de trabajo comunes a todo el litoral y prefirió tratar aisladamente los conflictos locales, procurando que no se extendieran las mejoras conseguidas en los puertos más combativos (Colectivo Xerminal, 1990).

En este contexto, el control sindical del puerto pesquero vigués, el más importante de la pesca de altura en el Estado junto al de Pasaia, fue la piedra de toque no solo para Galicia sino para el resto del litoral. Así, la CNT decidió el amarre de las parejas de Bouzas para el Primero de Mayo de 1931, aprovechando el final de la marea; y las reivindicaciones eran las que se comenzaban a negociar en Madrid. La relativa victoria sobre una Patronal cada vez más beligerante con las disposiciones laborales de la República, permitirá reforzar el sindicato tras su reconocimiento por parte de los armadores, lo que a su vez

8. Industrias Pesqueras, Vigo, 15/5/1931. 
contribuirá a la consolidación de un sindicalismo marinero coordinado a escala estatal. Consecuentemente, se acometió la inmediata creación de la referida Federación Nacional, a lo que también ayudó la firmeza y continuidad mostrada por El Despertar Marítimo coruñés; por contra, la huelga de los pescadores de Bouzas también puso de manifiesto la urgencia de reorganizar la Patronal española de la pesca de altura.

Volviendo a Bouzas, la aplicación de las nuevas bases de trabajo ${ }^{9}$ permitió practicar una presión en aumento sobre la contratación de los tripulantes y, de rebote, sobre la sindicación de los pescadores de otras villas de las Rías Baixas que descargaban o se enrolaban en el puerto vigués, tal y como ya se practicaba hacía tiempo en Coruña con otros puertos norteños, caso de la importante flota pesquera de Cariño. La integración en la CRG de veteranas sociedades marineras distribuidas por todo el litoral gallego, fue progresiva a partir del verano de 1931, un momento en el que la coyuntura política favorecía al sindicalismo y era patente la expansión de la pesca semi-industrial en la mayoría de las villas costeras. Las primeras en incorporarse fueron las de Porto do Son, Moaña, Cangas y Bueu, la mayoría procedente de la UGT, de manera que, en breve, la Confederación contará con sindicatos en casi todos los puertos del litoral gallego.

La moderación de la estrategia socialista, centrada en evitar problemas al Gobierno republicano y respaldar su política sociolaboral reformista, tuvo bastante que ver en este cambio de relación de fuerzas. Así, el delegado vigués en la Federación del Transporte, Pesca e Industrias Marítimas de España (UGT), criticó en agosto de 1933 el abandono de la huelga de los pescadores de Baiona llevada a cabo el año anterior; por toda respuesta, la Ejecutiva señaló que los tripulantes declararon el paro sin consultar y "dieron muestras de extremismos y cometieron atentados" ${ }^{\prime \prime}$. Como resultado, la UGT conservó muy poca afiliación entre los pescadores gallegos durante el período republicano; además, la central socialista resultó perjudicada por la escasa presencia de organismos de arbitraje gubernamentales (los Jurados Mixtos, sobre los que giraba en buena medida la táctica sindical de la UGT) en los principales puertos gallegos.

\section{LA FEDERACIÓN NACIONAL DE INDUSTRIA PESQUERA Y SUS DERIVADOS}

La continuidad organizativa de la FRGIP resultó determinante para el referido proceso de integración. El cambio de nombre allá por 1926 (de Marítima a Pesquera) no era intrascendente: reflejaba un cambio en su concepción. Ahora, los sindicatos federados acogerán solo las profesiones vinculadas a la pesca, tanto de tierra como de mar. En 1933 la visión integral de la industria se completó, al incorporarse los chóferes propietarios

\footnotetext{
9. Según las bases, los armadores podían elegir los tripulantes únicamente entre los afiliados al Sindicato y solo se reconocía el despido procedente.

10. Actas de la Federación del Transporte, Pesca e Industrias Marítimas de España (UGT), 15/1/1933, Cp. 1121, L. 350, Serie Madrid; Archivo Histórico Nacional (AHN), Sección Guerra Civil, Salamanca.
} 
de las camionetas que trasladaban pescado al interior, desde Coruña, Ribeira, Marín o Cariño. Estos trabajadores jugaron un rol importante a la hora de consolidar la CNT en los puertos antedichos, ya que su concurso fue decisivo a la hora de disuadir posibles descargas de pescado procedentes de esquiroles ${ }^{11}$.

Durante la Segunda República, la FRGIP se domicilió primero en Vigo y luego, a partir de 1934, en Marín; en las dos localidades asumirá la secretaría Manuel Fandiño, que actuó como liberado hasta la Guerra Civil. Como se dijo, la organización marinera gallega se adelantó a la implantación de las federaciones de industria decidida por la CNT en el Congreso celebrado en junio de 1931, puesto que ya en un Pleno de la CRG acontecido en octubre de 1930, Montes y Villaverde defendieron la creación de la Federación Nacional de Industria Pesquera y sus Derivados (FNIP) a partir de la FRGIP, ya perfilada desde hacía tiempo en sentido industrialista ${ }^{12}$. Así, la FRGIP fue la primera federación de industria de la CNT que funcionó en Galicia y posiblemente en el Estado español. En su andadura, los cuadros marineros agrupados en la CRG, asumieron la progresiva interacción existente entre los diversos puertos y la combinación entre lo local y la "globalización" que se derivaba del funcionamiento de los grandes nodos pesqueros; también tuvieron muy presentes la expansión de las nuevas modalidades pesqueras y el sesgo cada vez más diversificado de estas, asimiladas a la dinámica propia de un complejo industrial. Esta comprensión, permitió la formulación de una ajustada propuesta orgánica de carácter regional basada en la autonomía sindical, de manera que la FRGIP alcanzó una significativa implantación: alrededor de 12.500 asociados de 24 puertos en 1932 que, en Julio de 1936, ya se convirtieran en torno a 18.000 afiliados de ambos sexos, que engrosaban sindicatos radicados en 47 puertos distribuidos por toda la costa gallega. De hecho, la CRG tuvo su mayor presencia en el sector de la pesca, que agrupaba el $43 \%$ del total de su afiliación ${ }^{13}$.

La incorporación de las antiguas sociedades marineras a la CNT facilitó su progresiva conversión en Sindicatos de Industria Pesquera (SIP), que distribuían los diversos oficios en las respectivas Secciones. La transformación de la Sección Flota Pesquera del Sindicato General de Trabajadores vigués en Sindicato de Industria Pesquera "Mar y Tierra" poco antes del Congreso de 1931, inició las renovaciones organizativas: primero Marín y Coruña; luego, vendría la reforma de las sociedades existentes en Cangas, Moaña, A Guarda, Ribeira..., o la creación de sindicatos de industria allí donde no existía

\footnotetext{
11. CNT, Madrid, 18/10/1933.

12. Solidaridad Obrera, Coruña, 13/12/1930.

13. Solidaridad Obrera, Coruña, 3/9/1932; El Avance Marino, Pasaia, mayo 1936; Solidaridad Obrera, Barcelona, 5/5/1936.
} 
organización previa. Los Estatutos del SIP de la localidad de Espasante, indicaban en su artículo $5^{\circ}$ que:

“...estará compuesto de tantas secciones como características profesionales tenga la industria y sus similares, y el Sindicato reconocerá a cada una de ellas el derecho a tratar y resolver las cuestiones que no comprometan los intereses generales de la colectividad y cuando estos corrieran riesgo de ser comprometidos por medio de la declaración parcial o general de una huelga, la sección o secciones que se hallaran en tal caso no podrán declarar conflicto alguno sin previo conocimiento general de las demás secciones que integran el Sindicato" ${ }^{14}$.

Los sindicatos pesqueros más importantes fueron el SIP "Mar y Tierra" de Vigo-Bouzas, que en 1932 contaba con 2.200 socios; el de Coruña, con 2.370 afiliados/as; el de Marín, con 1.600; La Alianza Marinera de Cangas, 1.500 y con cifras parecidas la Fraternidad Marinera moañesa. Apuntaremos además, que en las antiguas sociedades de las villas marineras ahora remodeladas se mantuvo una apreciable presencia de pequeños armadores que patroneaban sus embarcaciones; volveremos brevemente sobre el tema.

Consecuentemente con su experiencia, como ya se dijo, se les debe a los marineros gallegos el impulso para la creación de la FNIP, partiendo de la propia organización territorial. En el proceso, tomó parte destacada el SIP de Vigo a través de su periódico Redención Marítima, editado a partir de junio de 1931 bajo la dirección de Manuel Fandiño. He ahí su tarjeta de visita en la prensa confederal:

“....En él se recogerán y propulsarán las aspiraciones de los obreros de todos los Sindicatos Marítimos de España, historiando sus movimientos reivindicativos y laborando por el estrechamiento más sólido de las relaciones, a fin de consolidar cuanto antes la Federación Nacional de Obreros de la Industria Pesquera y sus Derivados, cuya iniciativa fue unánimemente acogida por los delegados de los puertos españoles reunidos el pasado mes en Madrid...." ${ }^{15}$.

La FNIP se creó, pues, en la capital de España el 25 de octubre de 1931, coincidiendo su Congreso constituyente con la huelga de la flota de arrastre de Gijón y con el transcurso de las referidas negociaciones con la Patronal que, sin ninguna mejora efectiva, se estaban celebrando en Madrid. En correspondencia con la implantación confederal en el litoral galaico, el primer Comité de la FNIP residió en Coruña, ejerciendo como secretario liberado Manuel Montes, quedando la dirección del periódico Mar y Tierra a cargo de

14. Estatutos del Sindicato de la Industria Pesquera y sus Derivados de Espasante (Coruña), Archivo privado.

15. Solidaridad Obrera, Coruña, 27/6/1931. 
José Villaverde; en aquel momento, se declaraba una afiliación de 13.500 marineros $^{16}$. Un año más tarde, al acceder José Villaverde a la secretaría de la CRG, el Comité recalará en Vigo, siempre con Montes a la cabeza.

Durante todo el período republicano, el predominio de la organización galaica fue notorio dentro de la FNIP, pues en 1936 aquella representaba más del 40\% de sus miembros. En cualquier caso, no debería sorprendernos ni la faceta pionera en sentido organizativo, ni la importancia numérica de los trabajadores del mar gallegos, ya que la industria pesquera gallega era, con diferencia, la más desarrollada de toda la Península (Giráldez, 1996).

\section{PRÁCTICA SINDICAL}

Las sociedades marineras afectas a la FRGIP, caracterizadas por sus tácticas basadas en la acción directa y una gran autonomía, sostuvieron una marea de huelgas por toda la costa gallega, bien en puertos acostumbrados a ellas como Cangas, Moaña, Vigo, Coruña, Marín..., bien en otros en las que eran toda una novedad como Cariño, Celeiro, O Grove o Aldán.

En esta etapa republicana, las confrontaciones en la pesca serán las más prolongadas de todos los sectores de la economía gallega, debido a la postura cerrada mostrada por la Patronal, que debía afrontar la erosión del principio de autoridad tras la debilidad de las derechas en la alborada republicana y, al tiempo, el descenso de la tasa de ganancia resultante de la tendencia a la baja de los precios del pescado y del incremento de la presión sindical por unas mejores condiciones sociolaborales. Esta intransigencia se va a poner de manifiesto en las susodichas negociaciones de Madrid, que las tácticas dilatorias ejercidas por la delegación Patronal condujeron al fracaso más absoluto en la primavera de 1932 .

Mientras, la propia Federación de Armadores de Buques de Pesca de España se va a reestructurar en octubre de 1931, reforzando alianzas y procurando un mayor control de los conflictos locales. Así, se aceptará la entrada de algunos gremios independientes de armadores en distintos puertos, se adoptaron medidas solidarias con los armadores implicados en huelgas y se fijaron sanciones para quien no cumpliera los acuerdos. Además, a petición de los armadores de Cádiz, el Consejo de Dirección de la Federación, procedió a impartir "instrucciones a los puertos para las huelgas parciales, señalando las condiciones máximas y mínimas dentro de las que puedan resolver"17.

16. Constitución del Comité Nacional en Coruña, 23/11/1931; oficios FNIP, Sindicato General de la Industria Pesquera, AHN, Exp. Policiales H-13638, Años 1931-36.

17. Actas del Consejo de Dirección de la Federación de Armadores de Buques de Pesca de España, 20- 22 y $25 / 10 / 1931$. 
En este clima de confrontación y reforzamiento organizativo de las partes contendientes, destacó la resistencia de los pescadores, algo en lo que tuvo mucho que ver la referida posibilidad de faenar durante los conflictos con pequeñas lanchas y artes tradicionales propiedad de los huelguistas, su frecuente acceso a pequeñas parcelas de tierra en el litoral y la solidaridad de otros puertos vehiculizada por la FRGIP. En 1931, durante las huelgas de Teis, Baiona y Cangas un total de 2.040 marineros superaron las 200.000 jornadas de trabajo perdidas, si acreditamos en los datos del Ministerio de Trabajo, a menudo infravalorados. En la segunda mitad de 1932, el amarre de las parejas de Bouzas decidido por el SIP y que afectó a más de 3.000 tripulantes que reclamaban algunas cuestiones pendientes desde el año anterior en materia de descanso, incremento de las dotaciones y salarios, además de la Bolsa de Contratación gestionada por el sindicato, se demoró 5 meses (Colectivo Xerminal, 1990). El conflicto se desenvolverá no obstante en una coyuntura desfavorable para los marineros, caracterizada por la depreciación de las capturas, por una Patronal pesquera reforzada tras su reciente reestructuración y por unos sindicatos debilitados tras prolongadas huelgas que fueron resueltas en Huelva, Málaga, Algeciras, Cádiz y Gijón de manera contraria a los pescadores, en la mayoría de los casos.

Por tanto, tras soportar un masivo "lock out" (Bragado, 1933) a los dos meses de paro, el SIP "Mar y Tierra" de Vigo-Bouzas apenas pudo reaccionar cuando muchos armadores de Bouzas, amparados por la Federación de Armadores de Buques de Pesca de España, decidieron trasladar sus barcos al Sur de la Península tripulados por esquiroles procedentes de puertos sin organizar. En medio de violentos disturbios y a pesar de la importante solidaridad mostrada por la FRGIP, el SIP vigués perdió el control de las contrataciones en el muelle de Bouzas a manos de un sindicato inspirado por la Patronal, la Agrupación Independiente de Trabajadores de la Industria Pesquera. Así, una vez que los armadores incumplieron el laudo del Ministerio de Trabajo, por el que se daba por finalizada la huelga en diciembre de 1932 previa readmisión de los afiliados al SIP despedidos, la Agrupación hizo y deshizo a su gusto en los años siguientes.

El desgaste sufrido por la organización viguesa, tuvo dos importantes consecuencias. En primer lugar, la secretaría de la FRGIP recalará a partir de 1934 en Marín. En segundo, el Comité de la FNIP también abandonará Vigo a finales de 1933; la decisión se tomó en un Pleno Nacional de la Federación celebrado en Madrid el 9 de octubre de 1933. Allí asistieron delegaciones en representación de 11.966 asociados a diez sindicatos pesqueros de Galicia, tres de Asturias, uno de Euskadi, otro de Andalucía y dos, que asistieron con carácter informativo, de Barcelona. La baja de afiliación en relación al Congreso constituyente de la FNIP, dejaba traslucir una situación de crisis, cuyas causas reflejó el Comité en su Informe: 
“...La Federación se constituyó en octubre de 1931, y desde entonces puede decirse que toda su vida se desenvolvió con conflictos entre los trabajadores de la industria y la Patronal....Es innegable que los armadores al provocar sistemáticamente a los sindicatos pesqueros lo hacían previo meditado plan para abortar la organización naciente....Interesa declarar que las huelgas tuvieron un final lejos de nuestro deseo... En estas líneas explicativas de nuestros primeros pasos en la Federación encontrareis, compañeros delegados, la causa de que no se haya conseguido mayor efectividad en la labor encomendada a los que integramos este Comité..."18.

Además de las duras derrotas sindicales, que alentaron a la Patronal para dar la callada por respuesta a las reivindicaciones de la FNIP, había otras causas que explicaban el mal estado de la organización. De inicio, las divisiones dentro de la CNT, escindida entre los "moderados" nucleados en torno al "Manifiesto de los Treinta" y los partidarios de una confrontación abierta con el régimen republicano, división que provocó la marcha del SIP de Huelva y casi deshizo el propio Pleno, por el choque entre los dos sindicatos de Barcelona que se reclamaban de la FNIP. Como trasfondo estaba, además, la desconfianza que las federaciones de industria provocaban en no pocos confederales, que las consideraban limitativas para la autonomía de los sindicatos y caldo de cultivo de una posible burocracia sindical. En este sentido, creemos que no es casual el hecho de que tanto la FRGIP como la FNIP, se gestasen en una Confederación Regional (la CRG) que se alineó mayoritariamente con los "moderados" y en apoyo de tales federaciones (Pereira, 1994).

También las tensas relaciones con los socialistas causaron múltiples trifulcas durante los conflictos. Confrontados aquellos con las tácticas de los cenetistas y partidarios de los pactos con la Patronal a través de los organismos de arbitraje, en ocasiones reventaron los paros. Pasó, por ejemplo, en Cádiz en otoño de 1931, con La Naval (UGT) y El Despertar Marítimo (CNT) como contrincantes, situación que se repetirá en la citada huelga de las parejas viguesas, contribuyendo a la derrota de los marineros.

Para complicar las cosas, apareció un nuevo factor: las tentativas de los comunistas para controlar los sindicatos confederales. En Cádiz, una vez más, la presencia de $E l$ Despertar Marítimo en la organización sindical de carácter regional que intentó crear el PCE en Andalucía durante 1931 provocó que este sindicato gaditano fuera expulsado de la Federación Local cenetista y situado en "stand by" dentro de la FNIP hasta mediados de 1932, fecha en la que se desplazó a la dirección comunista y se reintegró a la CNT (Gutiérrez, 1994).

También los choques entre las diversas corrientes (socialistas, comunistas, libertarias), operantes en el SIP "La Unión Marítima" del puerto vasco de Pasaia, continuaron

18. CNT, 26/10/1933. 
después de la sangrienta huelga pesquera de $1931^{19}$. A lo largo del año siguiente, la FNIP envió su directiva a Pasaia para conseguir la adhesión del colectivo y tanto su secretario Manuel Montes como José Villaverde realizaron sendos actos de propaganda en Pasai San Pedro. Al final del segundo año republicano, tendrá lugar un referendum para decidir su integración, bien en la UGT, bien en la CNT; en la votación, los partidarios de la Confederación superaron a los de la UGT, pero los comunistas, defensores de la abstención puesto que pretendían declarar autónoma a La Unión, consiguieron que los abstencionistas fueran considerados vencedores, dándose el colectivo de baja en la UGT sin integrarse en la CNT. Esto provocó el abandono de los libertarios y la adhesión del sindicato a la Confederación General del Trabajo Unitaria, central comunista a la que estará vinculado hasta diciembre de 1935, fecha en la que retornará a la UGT (Pereira, 2010). Por consiguiente, a principios de 1933 se constituirá el SIP "El Avance Marino" integrado en la FNIP y domiciliado en Pasai San Pedro: durante el referido Pleno de la FNIP, el sindicato declaró una afiliación de 599 pescadores, rederos, empleados de la Pescadería y personal de la factoría de bacalao ${ }^{20}$.

Por último, a la FNIP tampoco le benefició la situación política al final del primer bienio republicano, porque importantes sindicatos como los de Cádiz y Pasaia tuvieron cerrados sus locales a partir del mes de mayo de 1933 por decisión gubernamental. Esta persecución interrumpió, de hecho, las tareas de propaganda del Comité de la FNIP al final de su mandato, dejando este inacabado. Como apuntaba José Villaverde desde Mar y Tierra:

“....Mucho se trabajó para agrupar a todos los que del mar viven en nuestros sindicatos. Pero es preciso fijar bien la atención en lo que falta por hacer. El Sur de España está casi huérfano de organización...El Mediterráneo, si bien cuenta con organización, ésta no está integrada a la Federación Nacional de Industria Pesquera. ....En el Cantábrico ocurre algo de lo mismo.... Se ha interrumpido esta obra de propaganda y es preciso organizarla, aunque ello cueste los mayores sacrificios. Los sindicatos de Galicia y Asturias deben pensar en ello inmediatamente...." ${ }^{21}$.

Pero en Galicia la organización no estaba para alegrías. Derrotas tan significativas como la sufrida en Bouzas, determinaron la posterior atonía de la organización viguesa, que además debió soportar las escisiones de algunas Secciones a favor de la UGT, y una estrategia concertada de los armadores de las Rías de Vigo y Pontevedra llevada a cabo durante los años 1933 y 1934 (Colectivo Xerminal, 1990). La susodicha estrategia,

19. En ese conflicto, declarado para reivindicar unas bases de trabajo parecidas a las que se negociaron en Madrid, hubo 7 muertos y 30 heridos a manos de la Guardia Civil.

20. Solidaridad Obrera, 25/2/33; CNT, 9/10/1933.

21. Mar y Tierra, Vigo, 10/6/1933. 
consistente en debilitar los sindicatos mediante la provocación de enfrentamientos derivados de posturas intransigentes, en las que el empleo del "lock out" y los esquiroles fue reiterado, se aplicó allí donde los colectivos de la FRGIP compartían espacio en tierra con la central socialista, caso de Vigo, Marín, Bueu o Cangas. En estos episodios, el abstencionismo de la UGT, la actuación de los "amarillos" y las escisiones inducidas acarrearon la desorganización societaria y la pérdida de afiliación en algunos puertos de las Rías Baixas.

En consecuencia, a finales de 1933, Asturias parecía la mejor solución para acoger al Comité de la FNIP, cuya secretaría radicó en Gijón y recayó con toda probabilidad en el directivo del SIP de aquella localidad, José A. López Iturralde. Pero el itinerario astur de la Federación duró poco, pues la represión desatada tras la Revolución de Octubre de 1934, obligará a trasladar el Comité a Cádiz, donde ocupará el cargo Julio Fernández. De todos modos, el paso de la FNIP por ambas localidades apenas dejó rastro, pues la precariedad del momento impidió continuar con la edición del periódico Mar y Tierra. En conclusión, en el otoño de 1935 la FNIP, lastrada por el poderío patronal, las divisiones y los problemas gubernativos, avanzó muy poco desde su creación. Según su Comité, si hubo algún crecimiento en aquel período (en Andalucía, en el Mediterráneo) se debía "al ambiente general y por los esfuerzos realizados por organizaciones ajenas a la industria"nz. Aún así, la FNIP era, en competencia con la Federación del Transporte, Pesca e Industrias Marítimas (UGT), de lejos, la propuesta sindical más implantada en las actividades pesqueras.

El asociacionismo representado en la FNIP tuvo hasta ese momento una identidad orientada a la resistencia frente a una realidad injusta, sin que esta visión fuese apenas compatible con otra de carácter más constructivo y cooperativo. Según el historiador Iago Santos Castroviejo, este fue un aspecto fracasado de las sociedades de clase, " $x a$ que un desenvolvemento reivindicativo sen un contrapeso de construcción das bases da producción autónoma dos traballadores é incompleto e desequilibrado" (Santos Castroviejo e Nores Soliño, 2005: 340). El propio Santos dejó, no obstante, bien claras las dificultades que conllevaba este talante cooperativo para los sindicatos confederales; he ahí el caso de Cangas:

“...a retribución á parte sostiña un sistema moi inxusto de distribución: os armadores estaban unidos coma unha piña a unha patronal de Vigo totalmente identificada con prestamistas, fabricantes, remitentes e vendedores que sostiñan sobre o prezo baixo do peixe os seus negocios. Polo tanto, os armadores á parte... admitían,..., o envilecimento do prezo do peixe e os altos prezos dos insumos en prexuizo dos intereses dos tripulantes... Os enfrontamentos entre armadores e tripulantes

22. El Avance Marino, abril 1936. 
son moi acedos en todo o período... 1914 a 1936,... e nestas circunstancias o programa de cooperación entre armadores e tripulantes...está permanentemente comprometido."(Santos Castroviejo e Nores Soliño, 2005: 344).

Con todo, la "FRGIP" desarrolló alguna experiencia que, basada en la propia capacidad organizativa, pretendió contribuir al ordenamiento pesquero: el Comité de Defensa de la Pesca del Litoral Gallego, por ejemplo, agrupó a delegados de las Rías Baixas y procuró colaborar con pequeños armadores que faenasen "a la parte" desde el verano de $1932^{23}$. El artículo $4^{\circ}$ de sus Estatutos, decía:

"Siendo la labor primordial de este Comité, la cooperación en sus múltiples aspectos, se admite el apoyo de los dueños de embarcaciones que estén de conformidad con estos postulados, y toda vez que ellos son coopartícipes en la pesca, al igual que a los marineros, pero permitiéndoles solamente el control en aquellas cuestiones que se refieren a la cooperación." 24.

Con sede en Vigo hasta octubre de 1934, tuvo como objetivos evitar la especulación en la venta de sardina y jurel; vigilar el empleo de dinamita en los barcos del cerco, multando a los infractores; eliminar intermediarios; efectuar en conjunto la compra de los insumos para la pesca; controlar producción y distribución para asegurar precios justos y no esquilmar el mar...

Fuera de Galicia, nos consta la colaboración de algunos sindicatos de pescadores de la costa catalana integrados en la FNIP, caso del Sindicato Único de Oficios Varios Sección Industria Pesquera de Roses (Girona), con el Montepio d'Obrers Pescadors de Catalunya, organismo de carácter cooperativo y mutualista creado por la Generalitat en la etapa republicana, para contrarrestar el carácter pro-patronal y centralista de los Pósitos de Pescadores (Cárdaba, 2001). Además, tenemos razones para pensar que, cuando se produce el golpe militar, en distintos territorios la organización marinera confederal estaba a favor de la incorporación de los Pósitos a la CNT, si bien desvinculados de los sindicatos, que velarían por la preservación de la orientación exclusivamente cooperativista de aquellos, ya en el campo de la producción, ya en el del consumo ${ }^{25}$.

23. El Pueblo Gallego, 13/4/1932.

24. Mar y Tierra, 19/4/1933.

25. Resoluciones del Congreso fundacional de la Federación Cantábrica de la Industria Pesquera, relativas a la "Posición de nuestra organización en relación a los Pósitos y a los trabajadores que en ellos trabajan", presentadas por los sindicatos de pescadores de Gijón, Candás y Santoña. Internationaal Instituut vor Sociale Geschiedenis Amsterdam (IISG), CNT Archives, 68B.4, “CNT AIT, F.N.I.P., Federación Cantábrica de la Industria Pesquera, Actas y Resoluciones del Congreso constitutivo celebrado en Santander los días 12 y 13 de Julio de 1936". Algo semejante acontecía en la CNT galaica (Pereira, 2012). 


\section{EL FRENTE POPULAR}

La impotencia de los sindicatos cenetistas de las Rías Baixas para resolver situaciones de conflicto sin el concurso de la UGT, alentó a la FRGIP en su firme defensa de las Alianzas Obreras "con finalidades concretas", postura manifestada durante el Pleno de la Federación celebrado en Santiago en el otoño de 1935. Allí, haciendo gala de un cierto pragmatismo (presente, asimismo, en la utilización sistemática de liberados y en el apoyo a la organización industrialista), diversos delegados plantearon el acuerdo con el sindicato socialista como base de la reorganización sindical en los puertos, tras la represión que siguió a la Revolución de Asturias:

"Molanes, de Cangas, demuestra lo fatal de la lucha entre trabajadores, que actuando en completo desacuerdo y divorcio, actúan desbarajustadamente, proporcionando el triunfo a las patronales. Cangas pertenece a la CNT, pero quiere que le ayuden, y por su parte ayudar a los demás en los conflictos entre el capital y el trabajo...."26.

Semejante posición en un clima de apasionado debate en el interior de la CNT sobre la conveniencia o no de tales Alianzas, es posible que originara tensiones entre la FRGIP y otros sindicatos integrados en la FNIP, que miraban con reticencia las propuestas de colaboración con la UGT. El desencuentro, acaso podría explicar parcialmente la pobre presencia de la FRGIP en el II ${ }^{\circ}$ Congreso de la FNIP, celebrado en mayo de 1936 en Zaragoza coincidiendo con el Congreso de la CNT, así como las críticas de algunos delegados allí presentes acerca del presunto desinterés y del pretendido localismo demostrado por la organización marinera galaica, en relación a la FNIP en general y al comicio en particular. En el curso de los debates, se aprobó un voto de censura a las delegaciones ausentes, en clara referencia a la $F R G I P^{27}$. No obstante, hay que tener en cuenta no solo la desorganización imperante en Galicia y Asturias debido a la represión post-Octubre de 1934, sino también la situación por la que pasaban en la primavera de 1936 los pescadores de la sardina, principal "clientela" de los sindicatos de las Rías gallegas, muy afectados económicamente por su depreciación y los paros masivos que tenían lugar, para entender la disminuida asistencia gallega a la capital maña.

La celebración del Congreso de la FNIP, tuvo bastante que ver con la presencia en Pasaia de un liberado muy experimentado, llamado Miguel González Inestal. De ascendencia salmantina y residencia madrileña, Inestal participara con anterioridad en la organización del potente Sindicato de la Construcción en la capital española y como redactor del periódico CNT (Iñíguez, 2001). En Pasaia, el salmantino asumió la secretaría del sindicato El Avance Marino, ejerciendo, además, como director de su periódico que, impreso a partir de noviembre de 1935, tomó idéntica denominación. De la mano de

26. Solidaridad, Coruña, 5/10/1935.

27. El Avance Marino, mayo 1936. 
Inestal, el vocero contribuyó a la revitalización de la FNIP, prácticamente desaparecida desde Octubre de 1934, alentando la celebración de un Congreso que la reorganizara a fondo. Celebrado en un contexto de impulso sindical tras la victoria del Frente Popular, en el Congreso estuvieron representados 39.260 afiliados, tres veces más que en la fecha de constitución de la FNIP. Por el momento se mantenía el predominio galaico, pero ya matizado por el importante medre de los sindicatos andaluces (que sobrepasaban el 30\% de la afiliación) y la expansión de la organización pesquera por el Cantábrico, Levante, Catalunya y Canarias (ver Tabla I).

Tabla 1.Afiliación a la "Federación Nacional de Obreros de la Industria Pesquera" (CNT). Registrada en el Congreso de Mayo 1936

\begin{tabular}{|l|c|c|c|c|}
\hline Comunidades & Afiliados & \%Total & Puertos & \%Total \\
\hline Galicia & 15620 & 40 & 37 & 47 \\
\hline $\begin{array}{l}\text { Andalucía y } \\
\text { Norte África }\end{array}$ & 12524 & 32 & 15 & 19 \\
\hline Euskadi & 991 & 3 & 2 & 2 \\
\hline Santander & 571 & 1 & 4 & 5 \\
\hline Asturias & 2800 & 7 & 5 & 7 \\
\hline Catalunya & 1700 & 4 & 4 & 2 \\
\hline Canarias & 2100 & 5 & 7 & 4 \\
\hline Levante & 2200 & 6 & 78 & \\
\hline Baleares & 650 & 2 & 3 & 2 \\
\hline Total & 39260 & & & 78 \\
\hline
\end{tabular}

Fuente: Elaboración propia a partir de: "El Avance Marino", Pasaia, n 7, Mayo 1936 y Congreso CNT, Mayo de 1936, "Solidaridad Obrera" (Barcelona), 5/5/36.

El evento retomó temas acordados en otras ocasiones y que no se pusieran en práctica debido a la azarosa trayectoria de la FNIP, como agrupar los sindicatos marineros del conjunto del litoral en Regionales, a imagen y semejanza de la FRGIP, y la unificación de las bases de trabajo para la pesca de arrastre. Así, si los armadores no respondían 
positivamente a partir del 15 de julio, la FNIP llamaría al amarre de toda la flota industrial el 1 de agosto de $1936^{28}$. Finalmente, se determinó que el Comité de la Federación retornase a Vigo, ocupando una vez más la secretaría el asturiano López Iturralde.

En correspondencia con lo decidido, una semana antes del movimiento militar se constituyó la Federación Cantábrica de la Industria Pesquera en Congreso celebrado en Santander, auspiciado por El Avance Marino. En el acontecimiento participaron sindicatos vascos (Pasaia, Erandio, Bermeo), astures (Candás, Avilés, Gijón, Cudillero, Luarca) y santanderinos (Santoña, Laredo, Santander) en representación de 5.706 afiliados. Al cabo, la guerra impidió que su Comité pudiera residir en Pasaia ${ }^{29}$.

En Bouzas, el SIP recuperó de manera fugaz su pulso con la Patronal de las parejas en mayo de 1936, contando ahora con el apoyo de la UGT. Los anarcosindicalistas exigieron, entonces, el reconocimiento del sindicato, el despido de los antiguos esquiroles embarcados y su sustitución por los confederados, retomando la reivindicación de la Bolsa de Trabajo controlada sindicalmente. Solventadas estas cuestiones y en paralelo a Santander, Pasaia, Algeciras y Cádiz, el SIP “Mar y Tierra” intentó plantear algunos temas pendientes desde 1932, como el descanso o las vacaciones retribuidas para absorber los parados $^{30}$. Por este motivo organizó el amarre de las parejas a principios de junio, una situación que se extendió hasta la Guerra Civil debido a las maniobras dilatorias de los armadores, interesados en prolongar los conflictos locales y así boicotear la previsible presentación de nuevas bases de trabajo aprobadas con carácter general por la FNIP y que se pretendían negociar una vez más con la Patronal española ${ }^{31}$.

Ante la sucesión de conflictos en la pesca de altura, el Gobierno del Frente Popular organizó en Madrid mediado junio de 1936 una Conferencia para discutir las condiciones de trabajo, convocándose a la FNIP, a la minoritaria Federación de la UGT y a la Federación Española de Oficiales de Marina Civil; por la Patronal fue llamada la Federación de Armadores. En medio de mutuas acusaciones de boicoteo, la Conferencia fracasó, haciéndole llegar los armadores al Gobierno la imposibilidad de aplicar las mismas bases de trabajo en todos los puertos $^{32}$. En esta situación de bloqueo, estalló el golpe militar.

28. El Avance Marino, marzo 1936.

29. “CNT-AIT. F.N.I.P. Federación Cantábrica de la Industria Pesquera. Actas y Resoluciones del Congreso Constitutivo celebrado en Santander los días 12 y 13 de Julio de 1936., CNT Archives, 68 B.4, IISG.

30. Industrias Pesqueras, 15/5/1936.

31. Circular no 3 FNIP, fechada en Vigo el 7/7/1936, incluida en la Causa 1415-37, Tribunal Militar IV, Marina; Industrias Pesqueras, 1/6/1936.

32. Industrias Pesqueras, 15/6/1936. 


\section{CODA}

En la FRGIP participó un grupo de cuadros experimentados, cuya preparación no solo sostuvo a los sindicatos de la pesca en una etapa donde la conflictividad en el sector llegó a cotas muy altas, sino que jugó un papel destacado dentro de la Confederación Regional Galaica de la CNT. En este sentido, el anarcosindicalismo marinero, potente en una región des-industrializada -aunque sí lo estaba en el sector pesquero- y periférica como Galicia, influyó de manera decisiva en la sorprendente implantación confederal (más de 40.000 afiliados/as en 1936) en un territorio cuya abrumadora mayoría social estaba compuesta por pequeños propietarios rurales, refractarios en su mayoría a los sindicatos de clase (Pereira, 1994).

En definitiva, los marineros gallegos fueron pioneros en la peculiar conformación identitaria de la FNIP que, a pesar de su dificultoso itinerario y las especiales características de la acción sindical en el mar, fue la única federación de industria de la CNT que existió en la práctica durante toda la República, reforzando su singularidad el hecho de que su implantación y organismos representativos estuvieran fuera de los principales núcleos confederales, caso de Cataluña, Levante, Aragón y, de manera parcial, Andalucía. En este tiempo, la FNIP se dotó de estructuras y estrategias específicas dentro de la Confederación, pues fue uno de los pocos organismos que contó antes de la Guerra Civil con liberados sindicales y alentó la incorporación de los autopatronos transportistas y la colaboración con pequeños armadores. En paralelo, la FNIP, en perpetua confrontación con la gran Patronal pesquera y conservera, intentó regular y homogeneizar las relaciones laborales en la pesca industrial en todo el litoral español, procurando superar dinámicas localistas. Por último, durante el Frente Popular y con una trayectoria interrumpida bruscamente por la contienda, la FNIP se extendió desde su reducto galaico y consiguió una significativa presencia en toda la costa española, siendo la organización más representativa del sindicalismo de clase en la pesca. 


\section{REFERENCIAS BIBLIOGRÁFICAS}

Bragado, D. (1933) Derivaciones y consecuencias del locaut pesquero de Vigo. Vigo: FRGIP. Calbetón, F. (1918) "Proyecto de organización de los pescadores libres para fines sociales", Primer Congreso de Estudios Vascos, Oñate, pp. 144-163.

Calo Lourido, F. (1978) La cultura de un pueblo marinero: Porto do Son, Santiago: Universidad de Santiago de Compostela.

Calo Lourido, F. (1996) Xentes do mar. Traballos, tradición e costumes, Vigo: A Nosa Terra.

Candía, J. e Ribeiro, A. "A vila mariñeira de Fisterra”, en Galván, A. (Coord.) Vivindo do mar. Antropoloxía da Pesca en Galicia, Santiago: Consellería de Presidencia, Xunta de Galicia, pp. 75-108.

Cárdaba, M. (2001) "La col. lectivitat de la indústria pesquera de Roses de Vicens Soler", AIEE Figueres, 34, pp. 497-541.

Carmona, X. (1998) "Igualdade e desigualdade nas pesquerías galegas do século XVIII", Grial, 102, pp. 216-226.

Colectivo Xerminal (1990) "Crise económica e loitas sociais na Galicia republicana: o conflito pesqueiro vigués de 1932" en AAVV O Movemento Obreiro en Galicia: catro ensaios. Vigo: Xerais, Vigo, pp.139-312.

Delgado Cendagortagalarza, A. (1998) Bermeo en el siglo XX: política y conflicto en un municipio pesquero vizcaíno (1912-1955). San Sebastián: Eusko Ikaskuntza.

Filgueira Valverde, J. (1946) Archivo de Mareantes. Pontevedra: ISM.

García Allut, A. (1989) “A vila mariñeira de Muxía”, en Galván, A. (Coord.) Vivindo do mar. Antropoloxía da Pesca en Galicia, Santiago: Consellería de Presidencia, Xunta de Galicia, pp. 29-71.

Giráldez Rivero, J. (1989) “Aproximaçom ao sector pesqueiro galego no primeiro terço do século XX”, “Agália”, monográfico n², pp. 7-32.

Giráldez Rivero, J. (1996) Crecimiento y transformación del sector pesquero gallego (18801936). Madrid: MAPA.

González Laxe, F. (1977) Estrutura da pesca costeira galega. Vigo: Galaxia.

Gutiérrez Molina, J. L. (1994) Crisis burguesa y unidad obrera. El sindicalismo en Cádiz durante la Segunda República. Madrid: FAL.

Íñiguez, M. (2001) Esbozo de una Enciclopedia histórica del anarquismo español, Madrid: FAL.

Maná, O. (1994) "La Pêche et les pecheurs à Marin (1770-1915)", Memoire de Maitrise, 
Besançon: Université de Franche-Comté.

Martínez, M. F. (1989) “A vila mariñeira da Guarda” en Galván, A. (Coord.) Vivindo do mar. Antropoloxía da Pesca en Galicia, Santiago: Consellería de Presidencia, Xunta de Galicia, pp.173-207.

Ministerio de Industria y Comercio (1933) Estadística de Pesca. Año 1932. Madrid.

Ministerio de Marina (1920) Boletín de Pescas. Madrid: Dirección General de Navegación y Pesca/Instituto Español de Oceanografía, Agosto-Diciembre.

Pereira, D. (1992) “Asociacionismo e conflitividade na Galiza mariñeira (1870-1936)" en Pereira, D. (Ed.) Os Conquistadores Modernos. Movemento Obreiro na Galicia de anteguerra, Vigo: A Nosa Terra, pp. 151-172.

Pereira, D. (1994) A CNT na Galicia (1922-1936), Santiago: Laiovento.

Pereira, D. (2002) "Proletariado e loita de clases na Galiza de anteguerra" en Constenla Bergueiro, G. e Domínguez Castro L. (Eds.) Tempos de sermos. Galicia nos séculos contemporáneos, Vigo: Universidade de Vigo, pp. 123-146.

Pereira, D. (2010) Loita de clases e represión franquista no mar (1864-1939). Vigo: Xerais. Pereira, D. (2012) “Os Pósitos de Pescadores: do Antigo Réxime á II ${ }^{a}$ República. Unha iniciativa conciliadora?". Minius, 20, en prensa.

Rodríguez Santamaría, B. (1916) Los pescadores del Norte y Noroeste de España. Su vida social y particular por provincias. Madrid: Imprenta Alemana.

Roig, E. “La pesca a Catalunya”, Enciclopedia de Catalunya, Ed. Barcino, Barcelona, 1927. Santos Castroviejo, I. e Nores Soliño, A. (2005) Historia de Cangas, 1900-1936. Unha ribeira de pescadores. Vigo: A Nosa Terra.

Vázquez Varela, J. M. (Coord.) (1990) El Hombre y el Mar en la costa Suroeste de Pontevedra, Pontevedra: Diputación Provincial de Pontevedra. 